\title{
MMC-HVDC: Simulation and Control System
}

\author{
Suman Debnath \\ Oak Ridge National Laboratory \\ Knoxville, TN 37932 \\ Email: debnaths@ornl.gov
}

\author{
Madhusudhan Chinthavali \\ Oak Ridge National Laboratory \\ Knoxville, TN 37932 \\ Email: chinthavalim@ornl.gov
}

\begin{abstract}
Simulation of modular multilevel converter (MMC) based high-voltage direct current (HVDC) systems assumes significance due to their growing popularity. It could assist with the design of hardware, control systems of MMC and HVDC networks, and power system topology. However, simulation of MMC-HVDC using existing software takes a long time due to the presence of a large number of states and non-linear devices. This paper presents an ultra-fast single- or multi-CPU simulation algorithm to simulate the MMC-HVDC system based on statespace models and using hybrid discretization algorithm with a relaxation technique that reduces the imposed computational burden. Using the developed simulation algorithm, a control system is developed for an MMC-HVDC system that reduces the switching losses in the system.
\end{abstract}

\section{INTRODUCTION}

The modular multilevel converter (MMC) has become an attractive topology in high-voltage direct current (HVDC) transmission systems due to its attractive features like modularity and scalability [1]. Simulation of MMC, therefore, assumes significance. A few simulation algorithms of MMCHVDC have been proposed in [2]-[6]. While the simulation algorithm in [3] does not consider the blocked-state of the MMC submodules (SMs), the algorithms in [5], [6] are susceptible to numerical oscillations and inaccuracy while simulating the blocked-state of the SMs. The simulation algorithms in [2] [6] use Dommel algorithm and model the devices like IGBTs, diodes, and thyristors with simple resistors or with voltagesources and resistors. However, the modeling of devices as ideal devices can greatly reduce the computational burden imposed without significant loss of accuracy. The loss of accuracy is minimal as the conduction and switching power losses in the MMC-HVDC systems are extremely small compared to the total power transmitted [7].

The MMC-HVDC system, when modeled using ideal device models, represents a non-linear non-autonomous switched system that can be deduced as a system of semi-explicit differential algebraic equations (DAEs) like the ones defined in [8]. The presence of diodes and thyristors in the MMCHVDC system introduce stiffness in the DAEs that require

This manuscript has been authored by UT-Battelle, LLC under Contract No. DE-AC05-00OR22725 with the U.S. Department of Energy. The United States Government retains and the publisher, by accepting the article for publication, acknowledges that the United States Government retains a non-exclusive, paidup, irrevocable, world-wide license to publish or reproduce the published form of this manuscript, or allow others to do so, for United States Government purposes. The Department of Energy will provide public access to these results of federally sponsored research in accordance with the DOE Public Access Plan(http://energy.gov/downloads/doe-public-access-plan).

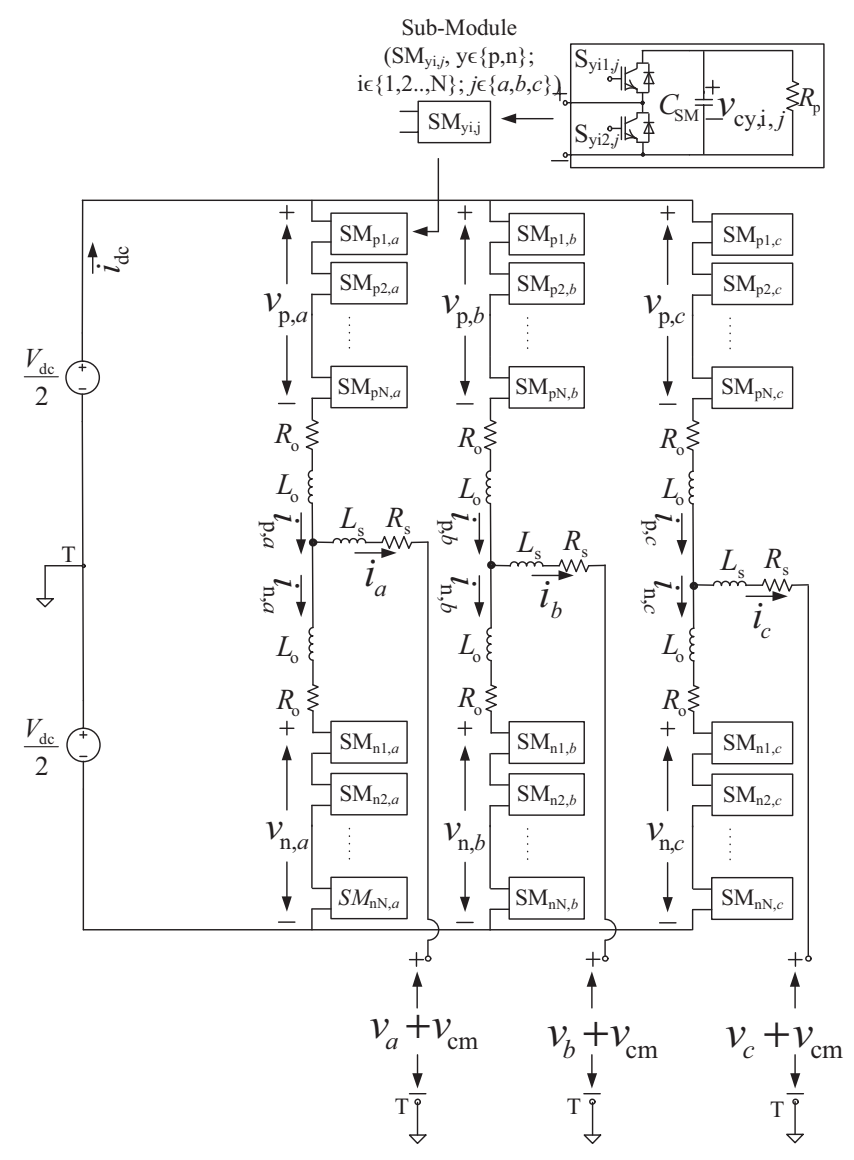

Fig. 1: Circuit diagram of MMC.

either explicit discretization algorithms with extremely small time-steps or implicit discretization algorithms with A-stable and stiff-decay properties [8]. Moreover, while modeling circuits with only ideal devices, state-space system models are generated for all the different circuit topologies possible based on the state of the diodes and thyristors [9]. This results in a large number of possible state-space system models for the MMC-HVDC system with the requirement of transition algorithms from one state-space system model to another based on an event detection algorithm [9]. The aforementioned requirements impose a large computational burden to simulate an MMC-HVDC system with a large number of devices.

In this paper, the MMC-HVDC system model is used to identify the components in the DAEs and the specific operating 


$$
\begin{gathered}
\left(L_{o}+L_{s}\right) \frac{\mathrm{d} i_{\mathrm{p}, j}}{\mathrm{~d} t}-L_{s} \frac{\mathrm{d} i_{\mathrm{n}, j}}{\mathrm{~d} t}=-\left(R_{o}+R_{s}\right) i_{\mathrm{p}, j}+R_{s} i_{\mathrm{n}, j}+\frac{V_{\mathrm{dc}}}{2}-v_{j}-v_{\mathrm{cm}}-v_{\mathrm{p}, j}, \forall j \in(a, b, c), \\
\left(L_{o}+L_{s}\right) \frac{\mathrm{d} i_{\mathrm{n}, j}}{\mathrm{~d} t}-L_{s} \frac{\mathrm{d} i_{\mathrm{p}, j}}{\mathrm{~d} t}=-\left(R_{o}+R_{s}\right) i_{\mathrm{n}, j}+R_{s} i_{\mathrm{p}, j}+\frac{V_{\mathrm{dc}}}{2}+v_{j}+v_{\mathrm{cm}}-v_{\mathrm{n}, j}, \forall j \in(a, b, c), \\
v_{\mathrm{y}, j}=\sum_{\mathrm{i}=1}^{N} v_{\mathrm{sm}, \mathrm{y}, \mathrm{i}, j}=\sum_{i=1}^{N}\left[S_{\mathrm{yi} 1, j} v_{\mathrm{cy}, \mathrm{i}, j}+\left\{\left(1-S_{\mathrm{yi} 1, j}\right)\left(1-S_{\mathrm{yi} 2, j}\right) v_{\mathrm{cy}, \mathrm{i}, j}\right\} \operatorname{sgn}\left(i_{\mathrm{y}, j}\right)\right], \forall \mathrm{y} \in(\mathrm{p}, \mathrm{n}), \forall j \in(a, b, c), \\
\operatorname{sgn}(x)=\left\{\begin{array}{ll}
0 & \text { if } \quad x<0 \\
1 & \text { if } \quad x>0,
\end{array} \quad v_{\mathrm{cm}}=\frac{1}{6} \sum_{j=a, b, c}\left(v_{\mathrm{n}, j}-v_{\mathrm{p}, j}\right), \quad \sum_{j=a, b, c} i_{\mathrm{p}, j}=\sum_{j=a, b, c} i_{\mathrm{n}, j}\right.
\end{gathered}
$$

conditions in which the stiffness is introduced. The stiff parts of the DAE are separated and a hybrid discretization algorithm is used to simulate the different parts of the DAE. The hybrid discretization algorithm consists of an implicit and an explicit discretization algorithm that discretize the appropriate parts of the DAE. Moreover, a relaxation algorithm is introduced to avoid the requirement of the large number of state-space system models and their corresponding transition and event detection algorithms. The proposed simulation algorithm of the MMC-HVDC systems is validated using a reference PSCAD/EMTDC model under various operating conditions. It is used to develop a control system for the MMC-HVDC system that reduces the switching losses in the system.

\section{Proposed MMC Algorithm}

The circuit diagram of a three-phase MMC is shown in Fig. 1. The basics of operation of the MMC is explained in detail in [1] and is not repeated here.

\section{A. The MMC System Model}

The dynamics of the arm currents in the MMC of Fig. 1 are given by (1), where $v_{\mathrm{sm}, \mathrm{y}, \mathrm{i}, j}$ is the output voltage of SM-i in arm-y, phase- $j$. It can be observed from (1d) that there are only five independent arm currents.

The dynamics of the SM capacitor voltages in the MMC of Fig. 1 are given by

$$
\begin{array}{r}
C_{S M} \frac{\mathrm{d} v_{\mathrm{cy}, \mathrm{i}, j}}{\mathrm{~d} t}=-\frac{v_{\mathrm{cy}, \mathrm{i}, j}}{R_{p}}+S_{\mathrm{yi} 1, j} i_{\mathrm{y}, j}+\left(1-S_{\mathrm{yi} 1, j}\right) \\
\times\left(1-S_{\mathrm{yi} 2, j}\right) i_{\mathrm{y}, j} \operatorname{sgn}\left(i_{\mathrm{y}, j}\right), \\
\forall \mathrm{y} \in(\mathrm{p}, \mathrm{n}), \forall j \in(a, b, c) .
\end{array}
$$

Equations (1) and (2) are the set of semi-explicit DAEs that represent the overall dynamics of the MMC. Under normal operating conditions when one of the devices in the SM is always turned ON, (1c) and (2) become

$$
\begin{gathered}
v_{\mathrm{y}, j}=\sum_{\mathrm{i}=1}^{N} S_{\mathrm{yi} 1, j} v_{\mathrm{cy}, \mathrm{i}, j}, \forall \mathrm{y} \in(\mathrm{p}, \mathrm{n}), \forall j \in(a, b, c), \\
C_{S M} \frac{\mathrm{d} v_{\mathrm{cy}, \mathrm{i}, j}}{\mathrm{~d} t}=-\frac{v_{\mathrm{cy}, \mathrm{i}, j}}{R_{p}}+S_{\mathrm{yi} 1, j} i_{\mathrm{y}, j}, \\
\forall \mathrm{y} \in(\mathrm{p}, \mathrm{n}), \forall j \in(a, b, c) .
\end{gathered}
$$

When an SM changes its state under normal operating conditions, it will be in blocked-state for a small duration (defined by dead-time). The impact of dead-time is neglected in (3) as it has been observed to be insignificant on the states of the MMC-HVDC system that is controlled using the nearest level control. Based on (1a), (1b), and (3), the dynamics of the arm current of the MMC is given by

$$
\mathrm{M}_{1} \frac{\mathrm{d} \boldsymbol{x}_{1}}{\mathrm{~d} t}=\mathrm{A} \boldsymbol{x}_{1}+\mathrm{B} \boldsymbol{s}+\mathrm{C} \boldsymbol{z}
$$

where

$\mathrm{M}_{1}=\left(\begin{array}{ccccc}L_{o}+L_{s} & -L_{s} & 0 & 0 & 0 \\ -L_{s} & L_{o}+L_{s} & 0 & 0 & 0 \\ 0 & 0 & L_{o}+L_{s} & -L_{s} & 0 \\ 0 & 0 & -L_{s} & L_{o}+L_{s} & 0 \\ -L_{s} & L_{s} & -L_{s} & L_{s} & L_{o}\end{array}\right)$,

$\mathrm{A}=-\left(\begin{array}{ccccc}R_{o}+R_{s} & -R_{s} & 0 & 0 & 0 \\ -R_{s} & R_{o}+R_{s} & 0 & 0 & 0 \\ 0 & 0 & R_{o}+R_{s} & -R_{s} & 0 \\ 0 & 0 & -R_{s} & R_{o}+R_{s} & 0 \\ -R_{s} & R_{s} & -R_{s} & R_{s} & R_{o}\end{array}\right)$

$$
\begin{aligned}
\mathrm{B} & =\left(\begin{array}{cccc}
0.5 & -1 & 0 & 0 \\
0.5 & 1 & 0 & 0 \\
0.5 & 0 & -1 & 0 \\
0.5 & 0 & 1 & 0 \\
0.5 & 0 & 0 & -1
\end{array}\right), \\
\mathrm{C} & =\left(\begin{array}{cccccc}
-5 / 6 & -1 / 6 & 1 / 6 & -1 / 6 & 1 / 6 & -1 / 6 \\
-1 / 6 & -5 / 6 & -1 / 6 & 1 / 6 & -1 / 6 & 1 / 6 \\
1 / 6 & -1 / 6 & -5 / 6 & -1 / 6 & 1 / 6 & -1 / 6 \\
-1 / 6 & 1 / 6 & -1 / 6 & -5 / 6 & -1 / 6 & 1 / 6 \\
1 / 6 & -1 / 6 & 1 / 6 & -1 / 6 & -5 / 6 & -1 / 6
\end{array}\right)
\end{aligned}
$$

$$
\begin{aligned}
\boldsymbol{s} & =\left(\begin{array}{llllll}
V_{\mathrm{dc}} & v_{a} & v_{b} & v_{c}
\end{array}\right)^{\mathrm{T}} \\
\boldsymbol{x}_{1} & =\left(\begin{array}{llllll}
i_{\mathrm{p}, a} & i_{\mathrm{n}, a} & i_{\mathrm{p}, b} & i_{\mathrm{n}, b} & i_{\mathrm{p}, c}
\end{array}\right)^{\mathrm{T}} \\
\boldsymbol{z} & =\left(\begin{array}{llllll}
v_{\mathrm{p}, a} & v_{\mathrm{n}, a} & v_{\mathrm{p}, b} & v_{\mathrm{n}, b} & v_{\mathrm{p}, c} & v_{\mathrm{n}, c}
\end{array}\right)^{\mathrm{T}} .
\end{aligned}
$$

Equation (4) is a linear ordinary differential equation (ODE) if $z$ is treated as an input variable. Similarly, the dynamics of the capacitor voltage represented by (3b) is a linear ODE if $i_{\mathrm{y}, j}$ is treated as an input variable. The aforementioned partition of the system by previous algorithms either used an artificial delay [6] or through nested fast and simultaneous 


$$
v_{\mathrm{cy}, \mathrm{i}, j}[k]=\left\{\begin{array}{l}
v_{\mathrm{cy}, \mathrm{i}, j}[k-1]\left(1-\frac{h}{R_{\mathrm{p}} C_{\mathrm{SM}}}\right)+i_{\mathrm{y}, j}[k-1] \cdot \frac{h}{C_{\mathrm{SM}}}, \text { if } \quad i_{\mathrm{y}, j}[k-1] \geq 0 \quad \text { and } \quad S_{\mathrm{yi} 2, j}[k-1]=0, \\
v_{\mathrm{cy}, \mathrm{i}, j}[k-1]\left(1-\frac{h}{R_{\mathrm{p}} C_{\mathrm{SM}}}\right)+i_{\mathrm{y}, j}[k-1] \cdot \frac{h}{C_{\mathrm{SM}}}, \text { if } \quad i_{\mathrm{y}, j}[k-1]<0 \quad \text { and } \quad S_{\mathrm{yi} 1, j}[k-1]=1, \\
v_{\mathrm{cy}, \mathrm{i}, j}[k-1]\left(1-\frac{h}{R_{\mathrm{p}} C_{\mathrm{SM}}}\right), \text { otherwise. }
\end{array}\right.
$$

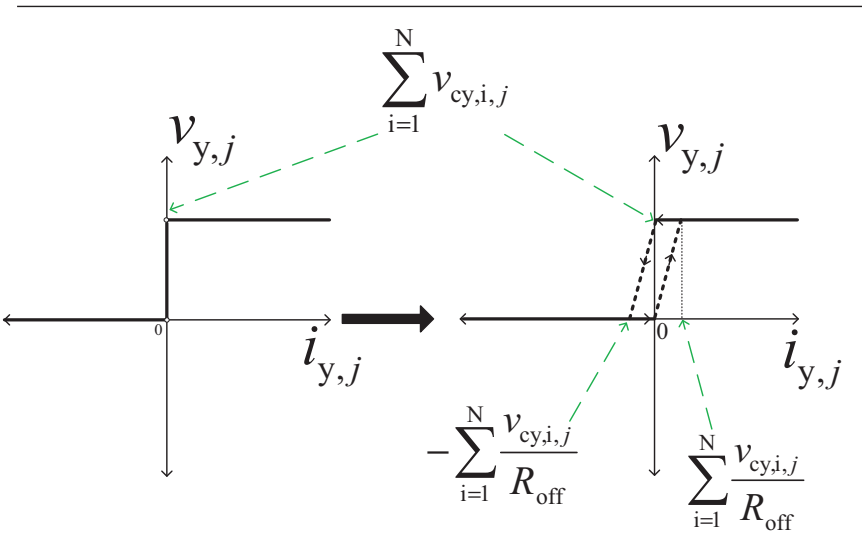

(a)

(b)
Fig. 2: Hysteresis relaxation technique: (a) arm voltage, and (b) approximated arm voltage.

simulation (NFSS) [2]-[5]. While the former usually leads to erroneous results with stiff systems as may be noticed in the MMC blocked-state results in [6], the latter imposes additional computational burden as will be shown later. The other means of partitioning the system is if at least one of the partitioned system can be discretized using an explicit algorithm, which is possible with both the partitioned systems in this case.

When the SMs are in blocked state, (1c) and (2) become

$$
\begin{gathered}
v_{\mathrm{y}, j}=\sum_{\mathrm{i}=1}^{N} v_{\mathrm{cy}, \mathrm{i}, j} \cdot \operatorname{sgn}\left(i_{\mathrm{y}, j}\right), \forall \mathrm{y} \in(\mathrm{p}, \mathrm{n}), \forall j \in(a, b, c), \\
C_{S M} \frac{\mathrm{d} v_{\mathrm{cy}, \mathrm{i}, j}}{\mathrm{~d} t}=-\frac{v_{\mathrm{cy}, \mathrm{i}, j}}{R_{p}}+i_{\mathrm{y}, j} \cdot \operatorname{sgn}\left(i_{\mathrm{y}, j}\right), \\
\forall \mathrm{y} \in(\mathrm{p}, \mathrm{n}), \forall j \in(a, b, c) .
\end{gathered}
$$

The $\operatorname{sgn}($.$) function in (7a) introduces stiffness in the system.$ The arm voltage $v_{\mathrm{y}, j}$ is defined by the system operating conditions and varies between 0 and $\sum_{i=1}^{N} v_{\mathrm{cy}, i, j}$ when $i_{\mathrm{y}, j}=0$, which represents an open arm condition. If the arm voltage is lesser than zero, then the lower diodes in the SMs of the arm turn ON. Similarly, if the arm voltage is greater than $\sum_{i=1}^{N} v_{\mathrm{cy}, i, j}$, then the upper diodes in the SMs of the arm turn ON. The variation of the arm voltage under the blocked scenario is shown in Fig. 2(a). To reduce the stiffness in the system and to avoid the requirement of circuit reconfiguration, a hysteresis relaxation technique is introduced that approximates the arm voltage as shown in Fig. 2(b). On substitution of the approximated arm voltages in (1a) and (1b) and using (1d), a linear ODE similar to (4) is obtained for the five independent arm currents. An argument similar to the one presented in the normal operation conditions scenario can be presented in the blocked SM scenario as well, which results in partitioning of the MMC-HVDC system in to two systems, namely, one representing the arm current dynamics, and the other representing the SM capacitor voltage dynamics. Each system is represented by linear ODEs with an input vector from the other system.

\section{B. Hybrid Discretization}

The greater the slope of the hysteresis curve in Fig. 2(b), the greater is the accuracy of the simulation of the MMCHVDC system under blocked SM scenario. However, a smaller time-step will be required to discretize the MMC-HVDC system model with explicit discretization algorithms. That is, for greater accuracy and to avoid unnecessarily small timesteps, implicit discretization algorithms with stiff-decay and A-stable properties, like backward Euler, should be preferred to discretize the MMC-HVDC system model.

Based on (7a), (1a), and (1b), it is noticed that the stiffness in the MMC-HVDC system model during the blocked SM scenario is only present in the arm current dynamics. The stiffness is introduced by the presence of $\operatorname{sgn}($.) function. The $\operatorname{sgn}\left(i_{\mathrm{y}, j}\right)$ present in the SM capacitor voltage dynamics can be treated as an input, thereby, making it a non-stiff system. Therefore, instead of discretizing the complete MMC-HVDC system model with an implicit discretization algorithm, only the arm current dynamics can be discretized using a stiff implicit discretization algorithm. The SM capacitor voltage dynamics can be discretized using an explicit discretization algorithm like forward Euler.

The use of implicit discretization algorithm on the complete MMC-HVDC system will require the inversion of $(6 N+5) \times$ $(6 N+5)$ matrix at every time-step, where $N$ is the number of SMs in each of the MMC. The variable $N$ can be of the order several hundreds today to several thousands in the near future. As will be shown in the next section, the hybrid discretization algorithm will require only the inversion of a $5 \times 5$ matrix at every time-step, thereby, reducing the computational burden imposed immensely.

\section{Implementation}

Forward Euler based discretization of the system representing the SM capacitor voltage dynamics results in (6), where $h$ is the simulation time-step and $k$ represents number of time-steps taken from $t=0$. Equation (6) is used in the SM capacitor voltage calculations under all operating conditions. The terms $\left(1-\frac{h}{R_{\mathrm{p}} C_{\mathrm{SM}}}\right)$ and $\frac{h}{C_{\mathrm{SM}}}$ can be calculated once at the beginning of the simulation and they 


$$
\begin{aligned}
& v_{\mathrm{y}, \mathrm{n}, j}[k]=\left\{\begin{array}{l}
0, \quad \text { if } \quad\left(i_{\mathrm{y}, j}[k-2]<0 \quad \text { and } \quad i_{\mathrm{y}, j}[k-1]<-\frac{\sum_{\mathrm{i}=1}^{N} v_{\mathrm{cy}, \mathrm{i}, j}[k]}{R_{\mathrm{off}}}\right), \\
0, \quad \text { if } \quad\left(i_{\mathrm{y}, j}[k-2]<0 \quad \text { and } \quad i_{\mathrm{y}, j}[k-1]>0\right) \quad \text { or } \quad\left(0<i_{\mathrm{y}, j}[k-1]<\frac{\sum_{\mathrm{i}=1}^{N} v_{\mathrm{cy}, \mathrm{i}, j}[k]}{R_{\mathrm{off}}}\right), \\
\sum_{\mathrm{i}=1}^{N} v_{\mathrm{cy}, \mathrm{i}, j}[k], \quad \text { otherwise. }
\end{array}\right.
\end{aligned}
$$

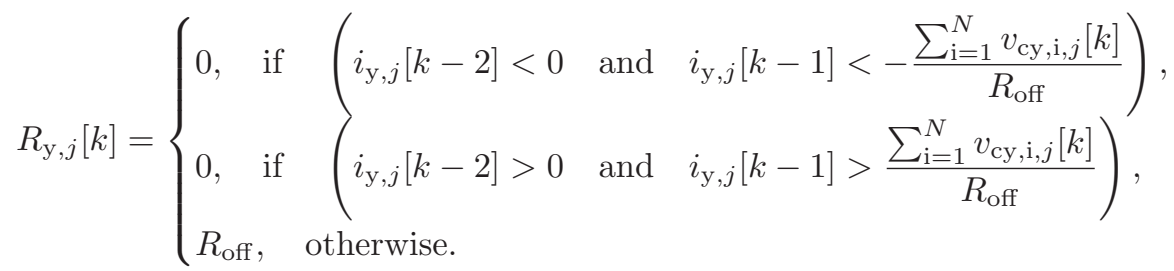

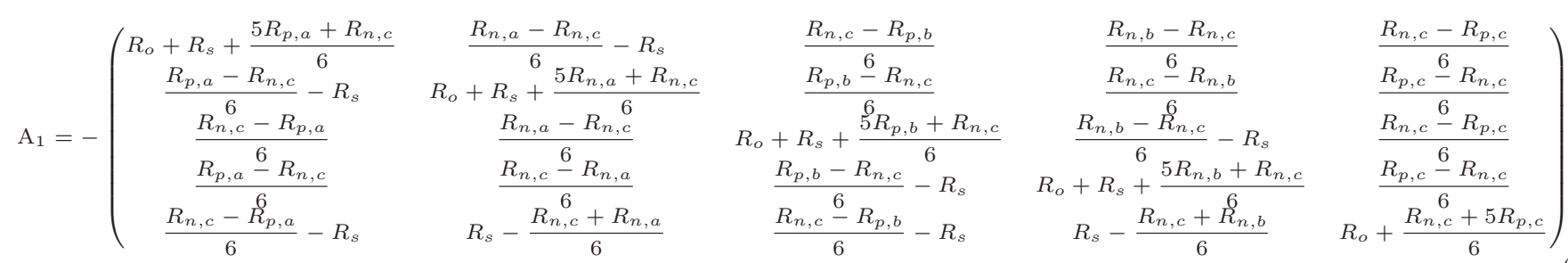

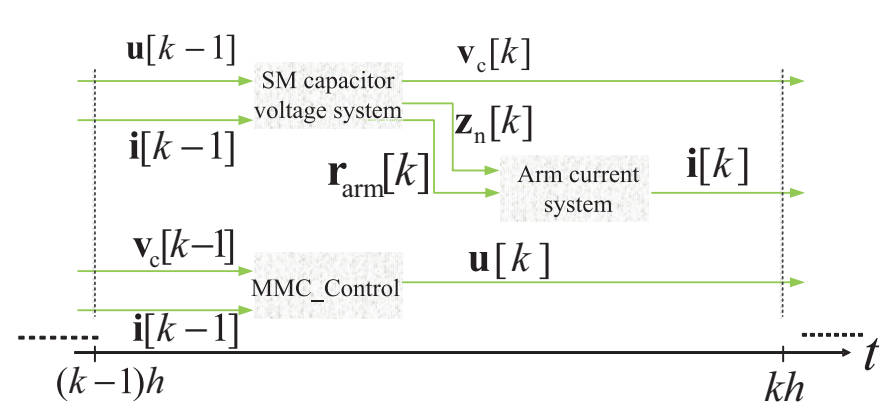

Fig. 3: Summary of MMC-HVDC simulation implementation.

remain the same thereafter. Therefore, only 3 floating-point operations are required to calculate each capacitor voltage in the worst-case. Backward Euler based discretization of the system representing the arm current dynamics results in the following:

$$
\begin{aligned}
\left(\mathrm{M}_{1}-\mathrm{A} . h\right) \boldsymbol{x}_{1}[k]= & \left(\mathrm{M}_{1}+\mathrm{A} \cdot h\right) \boldsymbol{x}_{1}[k-1]+h \cdot \mathrm{B} \boldsymbol{s}[k] \\
& +h . \mathrm{C} \tilde{\boldsymbol{z}}[k],
\end{aligned}
$$

where $\tilde{z}=\left(\begin{array}{llllll}\tilde{v}_{\mathrm{p}, a} & \tilde{v}_{\mathrm{n}, a} & \tilde{v}_{\mathrm{p}, b} & \tilde{v}_{\mathrm{n}, b} & \tilde{v}_{\mathrm{p}, c} & \tilde{v}_{\mathrm{n}, c}\end{array}\right)^{\mathrm{T}}$ and $\tilde{v}_{\mathrm{y}, j}$ is an approximated arm voltage.

The approximated arm voltage under blocked operating condition, as shown in Fig. 2(b), is implemented as follows:

$$
\tilde{v}_{\mathrm{y}, j}[k]=v_{\mathrm{y}, \mathrm{n}, j}[k]+i_{\mathrm{y}, j}[k] R_{\mathrm{y}, j}[k],
$$

where $v_{\mathrm{y}, \mathrm{n}, j}[k]$ and $R_{\mathrm{y}, j}[k]$ are defined in (8).

The approximated arm voltage under normal operating conditions is implemented as follows:

$$
\tilde{v}_{\mathrm{y}, j}[k]=v_{\mathrm{y}, \mathrm{n}, j}[k]+i_{\mathrm{y}, j}[k] R_{\mathrm{y}, j}[k],
$$

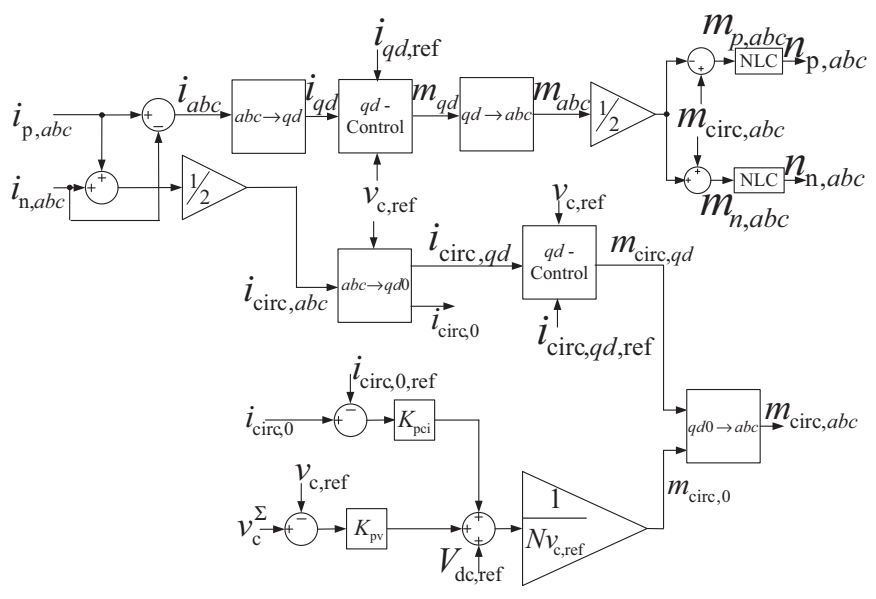

Fig. 4: Summary of MMC control system.

where

$$
\begin{aligned}
v_{\mathrm{y}, \mathrm{n}, j}[k] & =\sum_{i=1}^{N} S_{\mathrm{yi} 1, j}[k-1] v_{\mathrm{cy}, \mathrm{i}, j}[k], \\
R_{\mathrm{y}, j}[k] & =0 .
\end{aligned}
$$

Each approximated arm voltage requires only one floatingpoint operation per SM in the worst-case scenario as $S_{\mathrm{yi} 1, j}[k-$ 1] can be implemented using a conditional statement in (13a). That is, the SM capacitor voltage and arm voltage per SM calculation, under the worst-case scenario, requires only 4 floating-point operations. In comparison, the nodal algorithms [2], [4] require at least 25 floating-point operations [10]. That is, there is more than 6 times improvement in the theoretically required computational resources to evaluate the SM capacitor voltages and arm voltages. A comparison of the proposed 


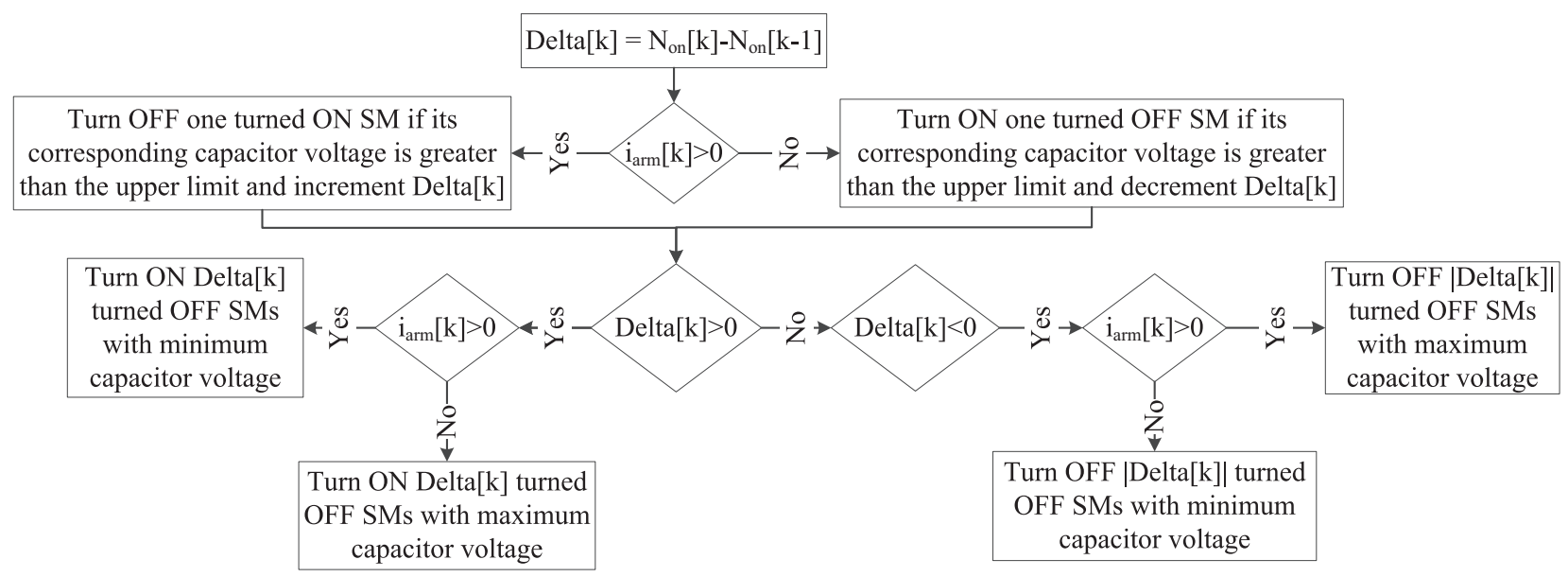

Fig. 5: MMC SM capacitor voltage balancing algorithm.

algorithm to the algorithms in [5], [6] is avoided due to their ineffective blocked-state implementations (although at least a two times improvement in theoretically required computational resources is observed).

Substituting the approximated arm voltages from (11) and (12) in (10) results in the following:

$$
\begin{gathered}
\left(\mathrm{M}_{1}-\mathrm{A}_{1} \cdot h\right) \boldsymbol{x}_{1}[k]=\left(\mathrm{M}_{1}+\mathrm{A}_{1} \cdot h\right) \boldsymbol{x}_{1}[k-1]+h \cdot \mathrm{B} \boldsymbol{s}[k] \\
+h \cdot \mathrm{C} \boldsymbol{z}_{\mathrm{n}}[k],
\end{gathered}
$$

where

$$
\boldsymbol{z}_{\mathrm{n}}=\left(\begin{array}{llllll}
v_{\mathrm{p}, \mathrm{n}, a} & v_{\mathrm{n}, \mathrm{n}, a} & v_{\mathrm{p}, \mathrm{n}, b} & v_{\mathrm{n}, \mathrm{n}, b} & v_{\mathrm{p}, \mathrm{n}, c} & v_{\mathrm{n}, \mathrm{n}, c}
\end{array}\right)^{\mathrm{T}},
$$

and $A_{1}$ is defined in (9). The inversion of $\left(\mathrm{M}_{1}-\mathrm{A}_{1} \cdot h\right)$, a $5 \times 5$ matrix, is required at every time-step to calculate the arm currents.

The overall implementation of the MMC-HVDC simulation algorithm is summarized in Fig. 3. In the figure, $\mathbf{v}_{\mathrm{c}}$ refers to the vector consisting of all the SM capacitor voltages, $\mathbf{r}_{\text {arm }}$ refers to the vector consisting of $R_{\mathrm{y}, j}$, and $\mathbf{i}$ refers to the vector containing all the arm currents. The SM capacitor voltage system calculations are based on (6) and the arm current system calculations are based on (14). Parallel computation of the SM capacitor voltages is feasible in the SM capacitor voltage system if a multi-core $\mathrm{CPU}$ is used.

\section{MMC CONTROL SYSTEM}

The MMC control system comprises $q d$ ac-side current control and $q d$ circulating current control of the second- and fourth-order harmonics. The ac-side current and circulating current control strategies are based on the strategies described in [11]. The control of fourth-order harmonic of the circulating current is not considered in [11], but is considered in this paper as third harmonic injection is considered in the modulation indices. The presence of third harmonic improves the range of modulation indices, but introduces a fourth harmonic component in the circulating current.
In addition to the $q d$ ac-side and circulating currents' control, 0-component of the circulating current and the average of all the SM capacitor voltages in the MMC (defined by $v_{\mathrm{c}}^{\Sigma}$ ) are controlled for better dynamic performance and for greater flexibility. The reference for $v_{\mathrm{c}}^{\Sigma}, v_{\mathrm{c}, \text { ref }}$, is varied based on the operating conditions so as to avoid saturation of the arm modulation indices $\left(m_{\mathrm{y}, j} \forall j \in(a, b, c), \mathrm{y} \in(\mathrm{p}, \mathrm{n})\right)$. Its derivation is shown below.

Defining $v_{j, \mathrm{c}}=\frac{v_{\mathrm{n}, j}-\dot{v}_{\mathrm{p}, j}}{2}$, the corresponding peak voltage is given by

$$
\begin{aligned}
v_{j, \mathrm{c}, \mathrm{pk}} & =\sqrt{v_{q, \mathrm{c}}^{2}+v_{d, \mathrm{c}}^{2}} / 1.15, \\
v_{q, \mathrm{c}} & =\left(v_{q}+\omega\left(L_{s}+\frac{L_{o}}{2}\right) i_{d, \mathrm{ref}}+\left(R_{s}+\frac{R_{o}}{2}\right) i_{q, \mathrm{ref}}\right), \\
v_{d, \mathrm{c}} & =\left(v_{d}-\omega\left(L_{s}+\frac{L_{o}}{2}\right) i_{q, \mathrm{ref}}+\left(R_{s}+\frac{R_{o}}{2}\right) i_{d, \mathrm{ref}}\right),
\end{aligned}
$$

where $v_{q}$ and $v_{d}$ are the $q d$ components, respectively, of the ac-side voltages $\boldsymbol{v}_{a b c}$. The rest of the parameters are described in Figs. 1 and 4. The factor of 1.15 is introduced due to the third harmonic injection in the modulation indices. The arm modulation index and its constraints are given by [1]

$$
-1 \leq m_{\mathrm{y}, j}=\frac{2 v_{j, \mathrm{c}}+V_{\mathrm{dc}}}{2 N v_{\mathrm{c}, \mathrm{ref}}} \leq 1 .
$$

The peak value of $m_{\mathrm{y}, j}$ is given by

$$
m_{\mathrm{y}, j, \mathrm{pk}}=\frac{v_{j, \mathrm{c}, \mathrm{pk}}+V_{\mathrm{dc}}}{2 N v_{\mathrm{c}, \mathrm{ref}}} .
$$

Substituting $v_{j, \mathrm{c}, \mathrm{pk}}$ from (16a) in (18) and re-aligning to obtain

$$
v_{\mathrm{c}, \mathrm{ref}}=\frac{2 v_{j, \mathrm{c}, \mathrm{pk}}+V_{\mathrm{dc}, \mathrm{ref}}}{2 N} .
$$

The reference for 0-component of the circulating current, 
$i_{\text {circ }, 0, \text { ref }}$, is given by

$$
i_{\text {circ }, 0, \text { ref }}=\frac{P_{\text {est }}}{3 V_{\mathrm{dc}, \text { ref }}},
$$

where

$$
P_{\mathrm{est}}=\frac{3}{2} v_{q} i_{q, \mathrm{ref}}
$$

The variation in the average value of the capacitor voltage reduces the switching frequency and increases efficiency of the system, as will be shown through simulation results. For stable operation of the MMC system, as has been noticed in simulations, the control of the 0-component of the circulating current assumes significance. The MMC control system is summarized in Fig. 4. NLC in Fig. 4 refers to nearest level control, which is described in detail in [12].

The SM capacitor voltage balancing algorithm is summarized in Fig. 5. It is based on the $O(N)$ algorithm developed in [11] to minimize the computational burden imposed. In addition to the algorithm in [11], an upper limit is applied on the SM capacitor voltages. If an ON-state SM has a capacitor voltage greater than the upper limit and current is positive, then it is turned OFF to avoid further charging of the corresponding capacitor. Similarly, if an OFF-state SM has capacitor voltage greater than the upper limit and current is negative, then it is turned $\mathrm{ON}$ to allow discharge of the corresponding capacitor. The upper limit is based on the design of the SM and depends on the lowest of the rated voltages of the different components of the SM. This improvement over the algorithm in [11] is necessary to avoid over-voltage conditions on the SM capacitors. Using this SM capacitor voltage balancing algorithm along with the variation in $v_{\mathrm{c}, \text { ref }}$ helps in maintaining the average switching frequency of each SM as close to the ac-side frequency as possible under various operating conditions.

\section{LOSS ANALYSIS}

The losses in the MMC are calculated based on losses in each IGBT and diode. The losses in each IGBT and diode are calculated by the sum of their corresponding conduction and switching losses. The conduction losses are calculated based on the product of the on-state voltage of the device and the current flowing through the device. The on-state voltage drop of each device is calculated based on

$$
v_{\mathrm{dev}, \mathrm{on}}=k_{1}+k_{2} i_{\mathrm{dev}}^{k_{3}},
$$

where $k_{1}, k_{2}$, and $k_{3}$ are the parameters obtained from curvefitting on-state voltage drop data from the device's datasheet and $i_{\mathrm{dev}}$ is the current flowing through the device. The subscript dev can be IGBT or diode based on the device being considered.

The switching losses are calculated based on dividing the energy losses in certain duration by the corresponding time duration. The energy losses can be the energy lost while turning on an IGBT, energy lost while turning off an IGBT, and energy lost in the reverse recovery of the diode. Each of aforementioned energies are calculated based on

$$
\begin{gathered}
E_{\mathrm{dev}, \mathrm{x}}=\frac{v_{\mathrm{dev}, \mathrm{off}}}{v_{\mathrm{nom}}}\left(E_{1, \mathrm{x}} i_{\mathrm{dev}}+E_{2, \mathrm{x}} i_{\mathrm{dev}}^{2}+E_{3, \mathrm{x}} i_{\mathrm{dev}}^{3}+E_{4, \mathrm{x}} i_{\mathrm{dev}}^{4}\right. \\
\left.+E_{5, \mathrm{x}} i_{\mathrm{dev}}^{5}\right),
\end{gathered}
$$

where $E_{1, \mathrm{x}}, E_{2, \mathrm{x}}, E_{3, \mathrm{x}}, E_{4, \mathrm{x}}$, and $E_{5, \mathrm{x}}$ are the parameters obtained from curve-fitting energy losses from the device's datasheet; $\mathrm{x}$ in the subscript $E_{\mathrm{dev}, \mathrm{x}}$ can be on, off, and rr to represent IGBT turn-on energy loss, IGBT turn-off energy loss, and diode reverse recovery loss, respectively; $v_{\mathrm{dev}}$,off refers to the voltage across the device during off-state; and, $v_{\text {nom }}$ refers to nominal voltage at which the energy data is available in the datasheet.

\section{Simulation Results \& COMPARison}

In this section, a real-world MMC system is considered to validate the accuracy of the developed simulation algorithm and compare the developed control system with the existing ones. A 401-level study MMC system is considered based on the France-Spain MMC-HVDC interconnection described in [4]. Only one MMC is considered to compare the developed simulation algorithms and the control system with existing algorithms and control systems, respectively. The dc-link is assumed to be a dc-source and the ac-side is assumed to be a 3-phase ac-source.

\section{A. Validation of Simulation Algorithm}
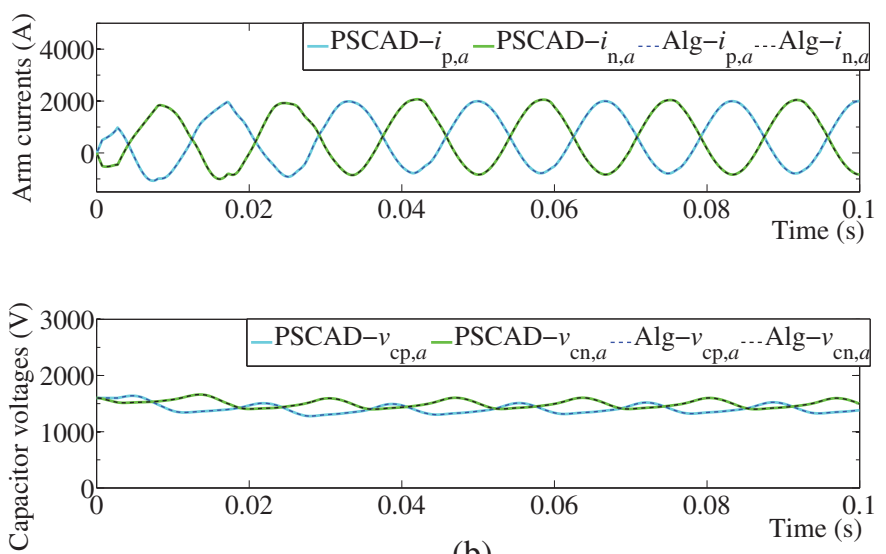

(b)

Fig. 6: MMC phase- $a$ states with $i_{q, \text { ref }}=2828$ A: (a) arm currents, and (b) average of arm SM capacitor voltages.

The reference results considered for validation of the proposed simulation algorithm are obtained from the detailed MMC model developed in PSCAD/EMTDC. Three casestudies are considered to compare the results obtained from the proposed simulation algorithm with the reference results: (i) steady-state operation, (ii) step-change in the $q$-axis current reference, and (iii) blocked-state operation under dc-link fault. For the third case-study, the dc-source voltage is changed to 0 to consider the worst-case scenario of the possible dc-link 

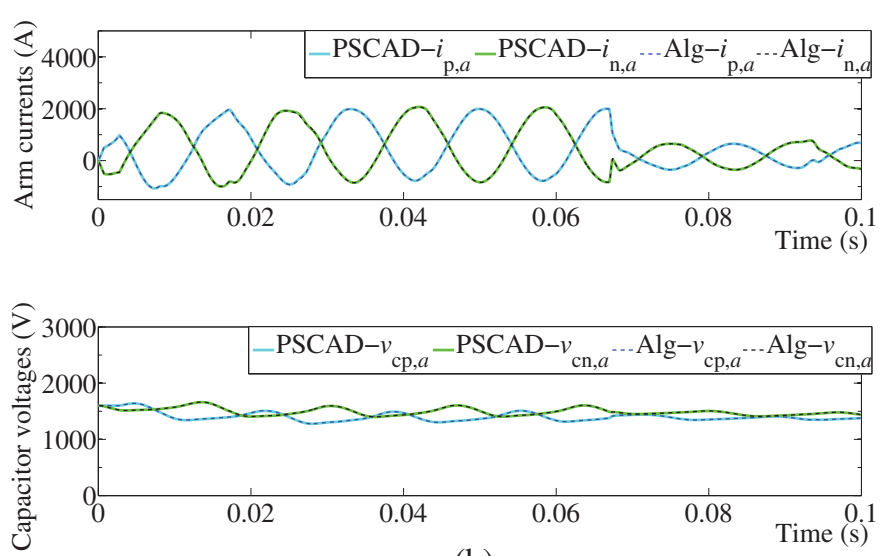

(b)

Fig. 7: MMC phase- $a$ states under step-change in $i_{q \text {,ref }}$ from 2828 A to 1000 A: (a) arm currents, and (b) average of arm SM capacitor voltages.

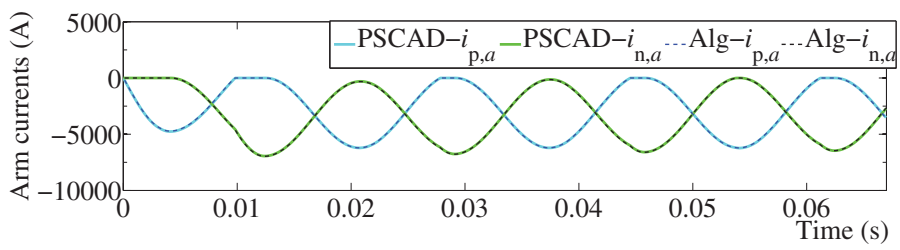

Fig. 8: MMC phase- $a$ arm currents in the blocked-state.

TABLE I: Loss Analysis Parameters

\begin{tabular}{|l|c|c|}
\hline Quantity & IGBT Value & Diode Value \\
\hline \hline$k_{1}$ & 0.0845 & $3.799 \times 10^{-3}$ \\
$k_{2}$ & 0.0679 & 0.141 \\
$k_{3}$ & 0.522 & 0.38 \\
$E_{1, \text { on }}$ & $1.9479 \times 10^{-3}$ & \\
$E_{2, \text { on }}$ & $-1.626 \times 10^{-6}$ & \\
$E_{3, \text { on }}$ & $1.4206 \times 10^{-9}$ & \\
$E_{4, \text { on }}$ & $-4.734 \times 10^{-13}$ & \\
$E_{5, \text { on }}$ & $5.804 \times 10^{-17}$ & \\
$E_{1, \text { off }}$ & $2.805 \times 10^{-3}$ & \\
$E_{2, \text { off }}$ & $-1.7955 \times 10^{-6}$ & \\
$E_{3, \text { off }}$ & $1.2902 \times 10^{-9}$ & \\
$E_{4, \text { off }}$ & $-4.125 \times 10^{-13}$ & \\
$E_{5, \text { off }}$ & $4.857 \times 10^{-17}$ & \\
$E_{1, \mathrm{rr}}$ & & $2.918 \times 10^{-3}$ \\
$E_{2, \mathrm{rr}}$ & & $-2.337 \times 10^{-6}$ \\
$E_{3, \mathrm{rr}}$ & \multicolumn{3}{|c}{} \\
$E_{4, \mathrm{rr}}$ & \multicolumn{2}{|c}{} \\
$E_{5, \mathrm{rr}}$ & & $-3.895 \times 10^{-9}$ \\
\hline
\end{tabular}

fault conditions. Once the fault occurs in the third case-study, the IGBTs are blocked.

The MMC phase- $a$ arm currents and average arm SM capacitor voltages are shown in Fig. 6 for the steady-state operation with the commanded $i_{q \text {,ref }}=2828 \mathrm{~A}$ and $i_{d, \text { ref }}=0$ A. The corresponding results for the step-change in $i_{q \text {,ref }}$ from 2828 A to 1000 A are shown in Fig. 7. The plots indicate that the results obtained from the proposed simulation algorithm

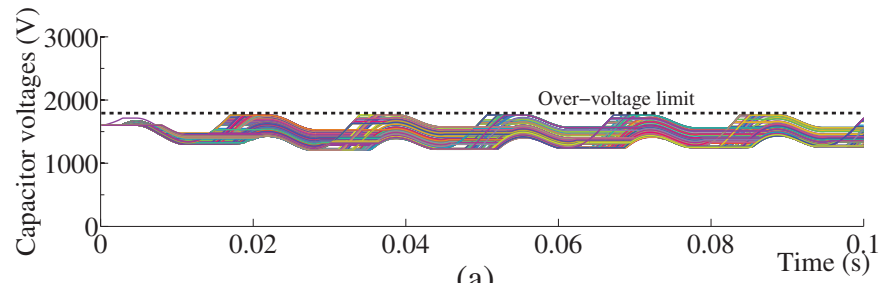

(a)

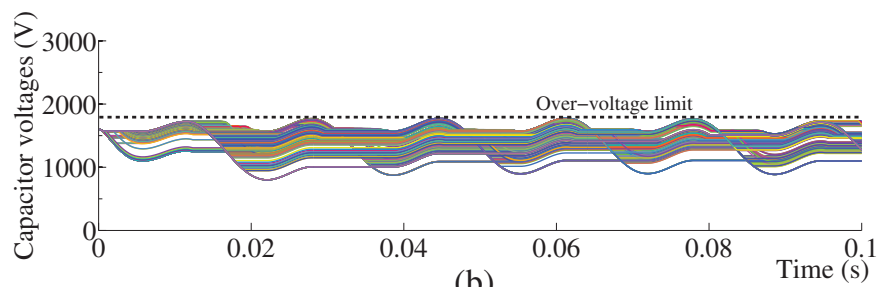

(b)
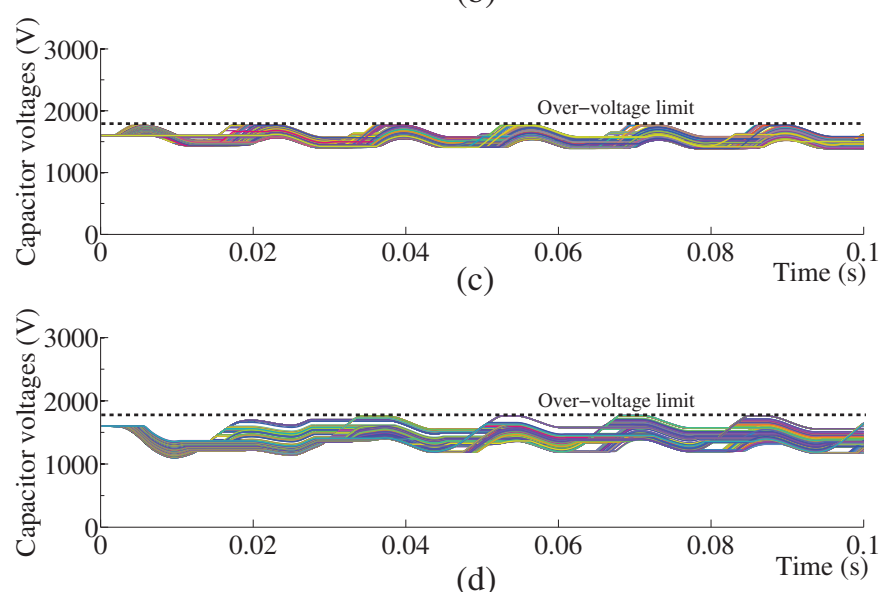

(d)

Fig. 9: MMC upper-arm phase- $a$ SM capacitor voltages with (a) $i_{q, \text { ref }}=2828 \mathrm{~A}, i_{d, \text { ref }}=0 \mathrm{~A}$, (b) $i_{q, \text { ref }}=-2828 \mathrm{~A}$, $i_{d, \mathrm{ref}}=0 \mathrm{~A}$, (c) $i_{q, \mathrm{ref}}=2000 \mathrm{~A}, i_{d, \mathrm{ref}}=1000 \mathrm{~A}$, and (d) $i_{q, \mathrm{ref}}=-2000 \mathrm{~A}, i_{d, \mathrm{ref}}=1000 \mathrm{~A}$.

closely match with the reference results. An error analysis performed in both the case-studies shows errors less than $0.5 \%$. The time taken by PSCAD/EMTDC to simulate $0.1 \mathrm{~s}$ is approximately 21 hours, whereas, the time taken to simulate 1 $\mathrm{s}$ using the developed simulation algorithm is approximately $3.8 \mathrm{~s}$. The time-step considered for simulation in both the cases is 4 us. The aforementioned result confirm the expected speedup of the simulation.

The MMC phase- $a$ arm currents in the blocked-state operation under dc-link fault are shown in Fig. 8. The plot indicates that the results obtained from the proposed simulation algorithm closely match with the reference results.

\section{B. Comparison of the Proposed Control System}

The operation of the proposed control system under various operating conditions and a comparison of the proposed control system with the existing ones are considered in this section. The comparison is performed only with control systems that consider an $O(N)$ SM capacitor voltage balancing algorithm like the ones in [13], [14]. These control systems impose much lower computational burden when compared to the ones that 
TABLE II: Energy Savings With Proposed Control System

\begin{tabular}{|c|c|c|c|c|}
\hline \multicolumn{2}{|c|}{ Operating Conditions } & \multicolumn{3}{c|}{ Energy Savings (W) } \\
\hline$i_{q, \text { ref }}(\mathrm{A})$ & $i_{d, \text { ref }}(\mathrm{A})$ & Algorithm in [13] & Algorithm in [14] & Modified Algorithm from [15] \\
\hline 2828 & 0 & 90238 & 6240898 & 1098568 \\
-2828 & 0 & 87933 & 6277300 & 4092670 \\
2000 & 1000 & 32777 & 5390646 & 3147586 \\
-2000 & 1000 & 20010 & 5341966 & 1042076 \\
\hline
\end{tabular}

consider an $O\left(N^{2}\right)$ or $O(N \cdot \log (N))$ SM capacitor voltage balancing algorithms like [15]-[18]. The algorithm in [11] is not considered for comparison due to capacitor voltage overvoltage violations that were observed.

For the loss analysis performed in this section, a $3.3 \mathrm{kV}$ and $1.5 \mathrm{kA}$ switch (IGBT with anti-parallel diode) with part number '5SNA1500E330305' is considered. The parameters defined in Section IV for the aforementioned device are documented in Table I.

With the over-voltage limit of SM capacitors set to $10 \%$ higher than the nominal capacitor voltage, the simulation of the study MMC system is performed using the developed control system. The individual upper-arm phase- $a$ SM capacitor voltages under four operating conditions are shown in Fig. 9 to show the successful operation of the proposed control system. The SM capacitor voltages show no over-voltage violations in all the cases.

A loss analysis is performed on the study MMC system under various operating conditions and using different control systems. The different control systems considered include the proposed control system in this paper and the ones described in [13]-[15]. The control system described in [15] is modified to an $O(N)$ SM capacitor voltage balancing algorithm. The energy savings from the proposed control system compared to the existing control systems is summarized in Table II. As may be noticed from the table, large energy savings ranging from $20 \mathrm{~kW}$ to $6.2 \mathrm{MW}$ are observed under the various operating conditions considered.

\section{Vi. CONCLusions}

A simulation algorithm of the MMC-HVDC based on statespace models, hybrid discretization, and a hysteresis relaxation technique is proposed in this paper that imposes lower computational burden compared to the existing simulation algorithms. Based on the developed simulation algorithm, a control system for the MMC-HVDC is proposed that lowers the switching losses over various operating conditions when compared to existing control systems. The simulation algorithm is validated using a reference PSCAD/EMTDC MMCHVDC system simulation results. Close to 6 times speed-up of the proposed simulation algorithm is proven theoretically with existing algorithms and close to 200,000 times speed-up when compared with the PSCAD/EMTDC reference simulation. The performance of the control system is verified through simulation and it is compared with existing control systems in terms of energy savings to indicate savings between $20 \mathrm{~kW}$ to $6.2 \mathrm{MW}$.

\section{REFERENCES}

[1] S. Debnath, J. Qin, B. Bahrani, M. Saeedifard, and P. Barbosa, "Operation, control, and applications of the modular multilevel converter: A review," IEEE Trans. Power Electron., vol. 30, no. 1, pp. 37-53, Jan 2015.

[2] U. Gnanarathna, A. Gole, and R. Jayasinghe, "Efficient modeling of modular multilevel hvdc converters (MMC) on electromagnetic transient simulation programs," IEEE Trans. Power Del., vol. 26, no. 1, pp. 316 324, Jan 2011.

[3] F. Yu, W. Lin, X. Wang, and D. Xie, "Fast voltage-balancing control and fast numerical simulation model for the modular multilevel converter," IEEE Trans. Power Del., vol. 30, no. 1, pp. 220-228, Feb 2015.

[4] H. Saad, S. Dennetiere, J. Mahseredjian, P. Delarue, X. Guillaud, J. Peralta, and S. Nguefeu, "Modular multilevel converter models for electromagnetic transients," IEEE Trans. Power Del., vol. 29, no. 3, pp. 1481-1489, June 2014.

[5] F. Ajaei and R. Iravani, "Enhanced equivalent model of the modular multilevel converter,' IEEE Trans. Power Del., vol. 30, no. 2, pp. 666673, April 2015.

[6] T. Maguire, B. Warkentin, Y. Chen, and J. Hasler, "Efficient techniques for real time simulation of mmc systems," in International Conference on Power Systems Transients, July 2013.

[7] S. Allebrod, R. Hamerski, and R. Marquardt, "New transformerless, scalable modular multilevel converters for HVDC-transmission," in IEEE Power Electronics Specialists Conference, June 2008, pp. 174179.

[8] U. Ascher and L. Petzold, Computational Methods for Ordinary Differential Equations and Differential-Algebraic Equations. Society for Industrial and Applied Mathematics, 1998. [Online]. Available: https://books.google.com/books?id=AqQ6QMthXkMC

[9] O. Wasynczuk and S. Sudhoff, "Automated state model generation algorithm for power circuits and systems," IEEE Trans. Power Syst., vol. 11, no. 4, pp. 1951-1956, Nov 1996.

[10] H. Saad, T. Ould-Bachir, J. Mahseredjian, C. Dufour, S. Dennetiere, and S. Nguefeu, "Real-time simulation of mmcs using cpu and fpga," IEEE Trans. Power Electron., vol. 30, no. 1, pp. 259-267, Jan 2015.

[11] Q. Tu, Z. Xu, and L. Xu, "Reduced switching-frequency modulation and circulating current suppression for modular multilevel converters," IEEE Trans. Power Del., vol. 26, no. 3, pp. 2009-2017, July 2011.

[12] Q. Tu and Z. Xu, "Impact of sampling frequency on harmonic distortion for modular multilevel converter," IEEE Trans. Power Del., vol. 26, no. 1, pp. 298-306, Jan 2011.

[13] Y. Li, E. Jones, and F. Wang, "The impact of voltage-balancing control on switching frequency of the modular multilevel converter," IEEE Trans. Power Electron., vol. 31, no. 4, pp. 2829-2839, April 2016.

[14] R. Chai, B. Zhang, Z. Hao, T. Zheng, S. Ma, and D. Chen, "A low switching frequency voltage balancing strategy of modular multilevel converter," in IEEE Region 10 Conference (TENCON), Oct 2013, pp. $1-4$.

[15] A. Hassanpoor, L. Angquist, S. Norrga, K. Ilves, and H.-P. Nee, "Tolerance band modulation methods for modular multilevel converters," IEEE Trans. Power Electron., vol. 30, no. 1, pp. 311-326, Jan 2015.

[16] F. Deng and Z. Chen, "A control method for voltage balancing in modular multilevel converters," IEEE Trans. Power Electron., vol. 29, no. 1, pp. 66-76, Jan 2014.

[17] A. Hassanpoor, A. Roostaei, S. Norrga, and M. Lindgren, "Optimizationbased cell selection method for grid-connected modular multilevel converters," IEEE Trans. Power Electron., vol. 31, no. 4, pp. 2780-2790, April 2016.

[18] J. Qin and M. Saeedifard, "Reduced switching-frequency voltagebalancing strategies for modular multilevel hvdc converters," IEEE Trans. Power Del., vol. 28, no. 4, pp. 2403-2410, Oct 2013. 